\title{
Initiation of therapy for obstructive sleep apnea syndrome: a randomized comparison of outcomes of telemetry-supported home-based vs. sleep lab-based therapy initiation
}

\author{
Ingo Fietze ${ }^{1}$ (D) Sebastian Herberger ${ }^{1} \cdot$ Gina Wewer $^{1} \cdot$ Holger Woehrle $^{2} \cdot$ Katharina Lederer $^{3} \cdot$ Aline Lips $^{2}$. \\ Leslee Willes ${ }^{4}$. Thomas Penzel ${ }^{1,5}$
}

Received: 18 November 2020 / Revised: 1 March 2021 / Accepted: 2 April 2021 / Published online: 15 May 2021

(C) The Author(s) 2021

\begin{abstract}
Purpose Diagnosis and treatment of obstructive sleep apnea are traditionally performed in sleep laboratories with polysomnography (PSG) and are associated with significant waiting times for patients and high cost. We investigated if initiation of auto-titrating CPAP (APAP) treatment at home in patients with obstructive sleep apnea (OSA) and subsequent telemonitoring by a homecare provider would be non-inferior to in-lab management with diagnostic PSG, subsequent in-lab APAP initiation, and standard follow-up regarding compliance and disease-specific quality of life.

Methods This randomized, open-label, single-center study was conducted in Germany. Screening occurred between December 2013 and November 2015. Eligible patients with moderate-to-severe OSA documented by polygraphy (PG) were randomized to home management or standard care. All patients were managed by certified sleep physicians. The home management group received APAP therapy at home, followed by telemonitoring. The control group received a diagnostic PSG, followed by therapy initiation in the sleep laboratory. The primary endpoint was therapy compliance, measured as average APAP usage after 6 months.

Results The intention-to-treat population (ITT) included 224 patients (110 home therapy, 114 controls); the per-protocol population (PP) included 182 patients with 6-month device usage data ( 89 home therapy, 93 controls). In the PP analysis, mean APAP usage at 6 months was not different in the home therapy and control groups $(4.38 \pm 2.04$ vs. $4.32 \pm 2.28, p=0.845)$. The prespecified non-inferiority margin (NIM) of $0.3 \mathrm{~h}$ /day was not achieved $(p=0.130)$; statistical significance was achieved in a post hoc analysis when NIM was set at $0.5 \mathrm{~h} /$ day $(p<0.05)$. Time to APAP initiation was significantly shorter in the home therapy group (7.6 \pm 7.2 vs. $46.1 \pm 23.8$ days; $p<0.0001)$.

Conclusion Use of a home-based telemonitoring strategy for initiation of APAP in selected patients with OSA managed by sleep physicians is feasible, appears to be non-inferior to standard sleep laboratory procedures, and facilitates faster access to therapy.
\end{abstract}

Keywords Obstructive sleep apnea - Automatic positive airway pressure C Continuous positive airway pressure $\cdot$ Compliance . Adherence

Ingo Fietze

ingo.fietze@ charite.de

1 Center for Cardiovascular Medicine, Interdisciplinary Center for Sleep Medicine, Charité-Universitätsmedizin Berlin, Luisenstraße 13, 10117 Berlin, Germany

2 Sleep and Ventilation Center Blaubeuren, Lung Center Ulm, Ulm, Germany

3 Advanced Sleep Research GmbH, Berlin, Germany

4 Willes Consulting Group, San Diego, CA, USA

5 Saratov State University, Saratov, Russian Federation

\section{Introduction}

Obstructive sleep apnea (OSA) is a common disorder, occurring at rate of up to $72 \%$ in the general population [1-3]. In addition to excessive daytime sleepiness, the presence of OSA also increases the risk of developing a number of important comorbidities, including hypertension, atrial fibrillation, coronary artery disease, stroke, diabetes and possibly even cancer [4-10].

Continuous positive airway pressure (CPAP) is the gold standard of treatment for OSA. The American Academy of Sleep Medicine (AASM) guidelines recommend CPAP or 
APAP for all patients with an apnea-hypopnea index (AHI) of

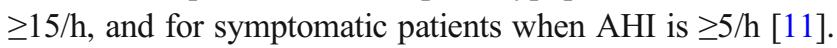
The benefits of CPAP therapy on OSA symptoms, concomitant conditions and quality of life cannot be realized if adherence to therapy is not achieved [12]. Adherence to CPAP therapy has therefore been widely studied, but there are many patient-related and patient-independent factors that influence long-term compliance [13-17].

The current clinical practice for starting CPAP or APAP therapy usually involves in-lab device initiation and titration. Laboratory-based polysomnography (PSG) is labor intensive and comparatively expensive, which could be a barrier for some patients. In addition, the high and increasing prevalence of OSA means that demand often exceeds capacity in sleep laboratories, resulting in long wait times [18]. Furthermore, access to sleep laboratories in some areas may be limited or non-existent.

Home-based respiratory polygraphy (PG) has been shown to be a reliable and cost-effective alternative to PSG for diagnosing sleep apnea [19-23] and is recommended by the AASM in patients without significant comorbidities [24]. Given the high prevalence of sleep apnea in Germany [3] and the long wait times for PSG at sleep laboratories, extending the use of PG and outpatient CPAP titration supported by telemonitoring managed by sleep physicians could provide a faster and easier access to therapy for selected patients and ease the burden of excessive demand at sleep centers.

This study investigated PAP therapy compliance of OSA patients, who had auto-titrating continuous positive airway pressure (APAP) therapy initiated in their home environment and were monitored by a homecare provider via telemonitoring, compared with the current practice of management including therapy initiation in a sleep laboratory and standard follow-up.

\section{Subjects and methods}

\section{Study design}

This randomized, open-label, parallel-group study was conducted at a single center in Germany with patient screening conducted over the period December 2013 to November 2015. The study protocol was approved by the institutional ethics committee. All patients gave written informed consent to participate in the trial, which was conducted in accordance with Good Clinical Practice and the Declaration of Helsinki.

\section{Patients}

Male and female patients aged 18-80 years who had moderate to severe OSA confirmed by ambulatory PG were eligible for the study. PG recordings were scored manually by a board- certified sleep physician. Exclusion criteria were as follows: mask intolerance; disability preventing independent nocturnal device usage; previous treatment for OSA (including uvulopalatopharyngoplasty surgery); modified Mallampati score of T3 or T4; participation in a clinical study within the previous 4 weeks; history of psychiatric, neurological or psychological conditions (excluding stable depression); clinically relevant lung or cardiovascular disease (excluding wellcontrolled hypertension, and treated cardiac arrhythmias or coronary heart disease); neuromuscular disease; history of cancer in the last 5 years; drug/alcohol abuse; and central sleep-related breathing disorders.

\section{Intervention groups}

Eligible patients who showed moderate to severe OSA (AHI $\geq 15 / h$ ) on 6-channel polygraphy (PG) and met all study inclusion criteria were randomized in a 1:1 ratio to the home management group or the standard of care group. The home therapy group received APAP initiation at home followed by telemetric monitoring; APAP pressure was set to 5-15 mbar for all patients.

The control group had APAP therapy setup and initiation in a sleep laboratory. In this group, patients underwent diagnostic PSG (1 night) and PSG-managed PAP therapy initiation (1-2 nights) in the sleep laboratory; PSG nights (two or three) occurred consecutively. Initial APAP pressure in the sleep laboratory was 5-15 mbar, which could be adjusted individually.

Telemetric monitoring in the home therapy group consisted in a weekly review of the wirelessly transmitted device usage statistics, including usage time, AHI (as measured by the device) and mask leakage by a specialist nurse in the homecare provider's data center. When usage data indicated problems, patients were contacted by phone by a service team member, who was also accessible to patients via a telephone hotline.

\section{Outcome measures}

The primary endpoint was average APAP usage (hours per night) at 6 months. Usage data for the past 6 months was retrieved from the device memory card. Secondary endpoints were the percentage of days with an APAP usage of $\geq 4 \mathrm{~h}$ and changes from baseline to 6 months in the following: Epworth Sleepiness Scale (ESS) score; total Functional Outcomes of Sleep Questionnaire (FOSQ) score; total Pittsburgh Sleep Quality Index (PSQI) score; and the apnea-hypopnea index (AHI). Additional outcome measures were oxygen desaturation index (ODI); numbers of obstructive, central and mixed events; and mean oxygen saturation $\left(\mathrm{SpO}_{2}\right)$ and leak. 


\section{Assessments}

Device usage was assessed and questionnaires completed at baseline, after 2 weeks of APAP therapy, and after 6 months of therapy. Respiratory parameters were determined using PG in both groups before study inclusion and after 6 months of APAP in both groups. In addition, the home management group was evaluated 2 weeks after treatment initiation. Patients in the control group underwent PSG at baseline and during APAP titration.

\section{Statistical analysis}

All analyses were conducted in the intention-to-treat (ITT) population (all patients randomized to treatment) and/or the per-protocol (PP) population (all patients who completed the 6-month follow-up visit and provided APAP usage data) using SAS version 9.4. Descriptive statistics (mean, median, standard deviation [6], standard error, minimum and maximum) were calculated for all continuous variables. Frequencies and percentages were calculated for categorical data. Baseline demographic and clinical characteristics were compared between treatment groups using descriptive statistics and compared using a 2 -sided $t$ test for continuous parameters and Fisher's exact test for categorical parameters. Data was controlled for normal distribution prior to further analysis, where applicable.

The primary endpoint (mean nightly APAP usage at 6 months) was assessed with a non-inferiority test by comparing the 2 treatment groups for all patients in the PP population using a non-inferiority 2-sample $t$ test at a one-sided significance level of $\alpha=0.025$. The planned non-inferiority margin (NIM) was $-0.3 \mathrm{~h} /$ day; a NIM of $-0.5 \mathrm{~h} /$ day was considered after a publication by the AASM highlighting that $0.5 \mathrm{~h}$ would be the margin for a clinically significant difference. [24] In addition, the between-group difference in mean usage was calculated along with the associated $95 \%$ confidence interval for the difference. A sensitivity analysis was conducted in a similar fashion on the ITT population using a NIM of $-0.5 \mathrm{~h} /$ day and assigning missing usage values to zero as a worst-case scenario. A subgroup analysis using ANOVA was conducted in the PP population to assess differences in mean APAP usage between treatment groups at 6 months in the following patient subgroups: by age ( $\leq 50$ vs. $>50$ years); male versus female; moderate OSA (baseline AHI $15 / \mathrm{h}$ to $<30 / \mathrm{h}$ ) versus severe OSA (baseline AHI $\geq 30 / \mathrm{h}$ ); and baseline ESS score $\geq 13$ versus baseline ESS score $<13$. Each ANOVA model included treatment group, patient subgroup and the interaction term as independent effects. $p$ values for the effect of the interaction between treatment group and subgroup are reported for each subgroup analysis.

All secondary analyses were generated as follows: device usage results at 6 months, including the proportion of days used, the proportion of days with usage $\geq 4 \mathrm{~h}$, mean usage and AHI were compared between groups in the PP population using a 2-sided $t$ test. The mean change from baseline to 6 months in ESS score, FOSQ score and PSQI score was compared using a 2-sided $t$ test, analyzing patients completing both baseline and 6-month questionnaires. A two-sided significance level of 0.05 was used for all secondary analyses.

\section{Results}

Of 505 patients initially screened, 224 patients with confirmed OSA were randomized to the home therapy $(n=110)$ or control $(n=114)$ groups and were included in the ITT population (Fig. 1). The PP population included 182 patients who had device usage reported at the 6-month visit (89 in the home therapy group and 93 in the control group) (Fig. 1); completion rates were similar in the two groups $(80.9 \%$ in the home therapy group and $81.6 \%$ in the control group). Four patients in the home therapy group and three in the standard of care group stopped using APAP but completed one or more of the sleepiness and sleep quality questionnaires. Seven patients in the home therapy group and eleven in the standard of care group discontinued the study between randomization and the week 2 visit, and another fourteen in the home therapy group and ten in the standard of care group discontinued between week 2 and 6 months. Two patients required a switch to CPAP or bilevel positive airway pressure. Patient clinical and demographic data at baseline are shown in Table 1 . The home therapy and control groups were similar at baseline. Mean follow-up time was $194.6 \pm 38.6$ days (range 62-336) in the home therapy group and $186.6 \pm 36.3$ (range $112-384$ ) in the control group.

\section{Device usage}

In the PP population, mean APAP device usage at 6 months was similar in the home therapy and control groups at just over $4 \mathrm{~h} /$ day $(4.38 \pm 2.04$ vs. $4.32 \pm 2.28$, respectively, $p=0.845)$. Statistical significance for the initially pre-defined non-inferiority margin of $-0.3 \mathrm{~h} /$ day was not achieved $(p=0.130)$. However, given the later published clinically important difference of $0.5 \mathrm{~h}$ as an important compliance difference by the AASM [25], statistical significance for better compliance in the home management group was achieved for a NIM of -0.5 $\mathrm{h} /$ day $(p=0.041)$ (Table 2$)$. In a sensitivity analysis based on the ITT population imputing missing values as the worst case (0 usage) and using a NIM of $0.5 \mathrm{~h} /$ day, statistical significance was not achieved ( $p=0.067$ ) (Table 2). Overall, we did not find any advantage of one treatment approach over the other across any pre-defined patient subgroup analyzed (Table 3). 
Fig. 1 Study flow chart. Completed $=$ having some device usage registered

Table 1 Patient demographic and clinical characteristics at baseline (intention-to-treat population)

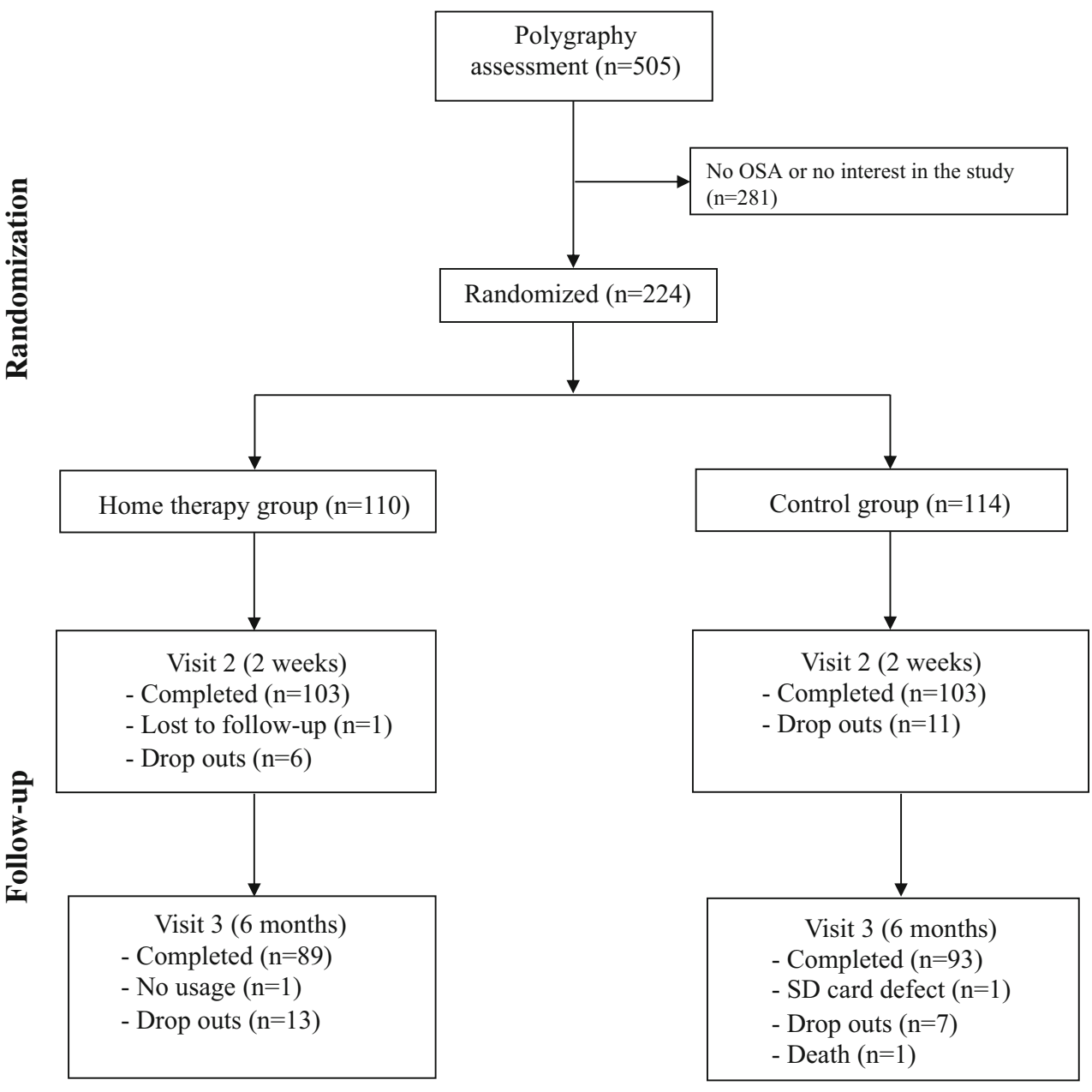

\begin{tabular}{|c|c|c|c|}
\hline & $\begin{array}{l}\text { Home therapy group } \\
(n=110)\end{array}$ & $\begin{array}{l}\text { Control group } \\
(n=114)\end{array}$ & $p$ value \\
\hline Age, years & $53.6 \pm 11.8(24-80)$ & $53.1 \pm 10.6(28-73)$ & 0.75 \\
\hline Male, $n(\%)$ & $90(83)$ & $90(79)$ & 0.50 \\
\hline Caucasian ethnicity, $n(\%)$ & $110(100)$ & $113(99)$ & 1.00 \\
\hline BMI, $\mathrm{kg} / \mathrm{m}^{2}$ & $32.8 \pm 6.4(21.7-51.7)$ & $31.7 \pm 5.6(21.3-49.5)$ & 0.17 \\
\hline AHI, $/ \mathrm{h}$ & $35.3 \pm 17.6(15.1-110.0)$ & $37.0 \pm 20.3(15.4-108.5)$ & 0.50 \\
\hline ODI, $/ \mathrm{h}$ & $29.9 \pm 18.6(0.3-92.1)$ & $34.5 \pm 21.8(3.8-112.0)$ & 0.09 \\
\hline Mask type, $n(\%)$ & & & 0.22 \\
\hline Face & $19(17.3)$ & $29(25.4)$ & \\
\hline Nasal & $48(43.6)$ & $39(34.2)$ & \\
\hline Nasal pillows & $43(39.1)$ & $46(40.4)$ & \\
\hline Mean $\mathrm{SpO}_{2}, \%$ & $91 \pm 3(72-95)$ & $90 \pm 3(78-95)$ & 0.53 \\
\hline Minimum $\mathrm{SpO}_{2}, \%$ & $76 \pm 9(51-91)$ & $74 \pm 9(51-86)$ & 0.08 \\
\hline ESS score & $11.2 \pm 5.2(1-24)$ & $10.9 \pm 4.9(2-24)$ & 0.64 \\
\hline PSQI score & $9.3 \pm 9.8(0-91)$ & $8.1 \pm 3.4(2-16)$ & 0.22 \\
\hline FOSQ score & $87.8 \pm 21.9(11-120)$ & $86.8 \pm 20.0(22-120)$ & 0.74 \\
\hline
\end{tabular}

Values are mean \pm standard deviation (range), or number of patients (\%)

$A H I$ apnea-hypopnea index, BMI body mass index, ESS Epworth Sleepiness Scale, FOSQ Functional Outcomes of Sleep Questionnaire, $P S Q I$ Pittsburg Sleep Quality Index, $\mathrm{SpO}_{2}$ oxygen saturation 
Table 2 Device usage analysis at 6 months

\begin{tabular}{llll}
\hline Visit 3 (6 months) & $\begin{array}{c}\text { Home therapy group } \\
\text { Usage (h/day) }\end{array}$ & $\begin{array}{l}\text { Control group } \\
\text { Usage (h/day) }\end{array}$ & Between-group difference (h/day) \\
\hline PP population & $(n=89)$ & $(n=93)$ & \\
Mean \pm SD & $4.38 \pm 2.04$ & $4.32 \pm 2.28$ & $0.06 \pm 2.17$ \\
$(95 \%$ CI) & $(3.95,4.81)$ & $(3.85,4.79)$ & $(-0.57,0.70)$ \\
Median (range) & $4.40(0.10-9.58)$ & $4.58(0.00-8.07)$ & \\
NIM -0.3 h/day & & & \\
NIM -0.5 h/day & & $(n=114)$ & $0.02 \pm 2.59$ \\
Sensitivity analysis* & $(n=110)$ & $3.52 \pm 2.66$ & $(-0.66,0.70)$ \\
ITT population & $3.54 \pm 2.52$ & $(3.03,4.02)$ & \\
Mean \pm SD & $(3.07,4.02)$ & $3.85(0.0-8.1)$ & \\
$(95 \%$ CI) & $3.76(0.0-9.6)$ & & 0.041 \\
Median (range) & & & \\
NIM -0.5 h/day & & & \\
\hline
\end{tabular}

* Missing mean usage imputed as zero

$C I$ confidence interval, $h$ hours, ITT intention-to-treat, NIM non-inferiority margin, $P P$ per-protocol, $S D$ standard deviation

\section{Secondary endpoints}

Device data at 6 months showed similar usage in terms of proportion of days used and proportion of days with usage

Table 3 Device usage at 6 months in patient subgroups (per-protocol population)

\begin{tabular}{|c|c|c|c|}
\hline Patient subgroup & $\begin{array}{l}\text { Home therapy group } \\
\text { Usage (h/day) }\end{array}$ & $\begin{array}{l}\text { Control group } \\
\text { Usage (h/day) }\end{array}$ & $p$ value* \\
\hline Age $\leq 50$ years & $\begin{array}{l}(n=31) \\
4.25 \pm 2.11 \\
(3.48,5.03)\end{array}$ & $\begin{array}{l}(n=38) \\
4.08 \pm 2.34 \\
(3.32,4.85)\end{array}$ & 0.76 \\
\hline Age $>50$ years & $\begin{array}{l}(n=58) \\
4.45 \pm 2.02 \\
(3.92,4.98)\end{array}$ & $\begin{array}{l}(n=55) \\
4.48 \pm 2.25 \\
(3.87,5.09)\end{array}$ & \\
\hline Male & $\begin{array}{l}(n=75) \\
4.47 \pm 2.07 \\
(3.99,4.94)\end{array}$ & $\begin{array}{l}(n=76) \\
4.43 \pm 2.18 \\
(3.94,4.93)\end{array}$ & 0.91 \\
\hline Female & $\begin{array}{l}(n=14) \\
3.93 \pm 1.93 \\
(2.81,5.05)\end{array}$ & $\begin{array}{l}(n=17) \\
3.80 \pm 2.71 \\
(2.40,5.19)\end{array}$ & \\
\hline Moderate OSA & $\begin{array}{l}(n=44) \\
3.91 \pm 1.97 \\
(3.31,4.51)\end{array}$ & $\begin{array}{l}(n=44) \\
4.26 \pm 2.04 \\
(3.64,4.88)\end{array}$ & 0.20 \\
\hline Severe OSA & $\begin{array}{l}(n=45) \\
4.84 \pm 2.03 \\
(4.23,5.45)\end{array}$ & $\begin{array}{l}(n=49) \\
4.37 \pm 2.50 \\
(3.65,5.09)\end{array}$ & \\
\hline Baseline ESS score $<13$ & $\begin{array}{l}(n=56) \\
4.33 \pm 2.12 \\
(3.77,4.90)\end{array}$ & $\begin{array}{l}(n=55) \\
4.40 \pm 2.39 \\
(3.76,5.05)\end{array}$ & 0.62 \\
\hline Baseline ESS score $\geq 13$ & $\begin{array}{l}(n=31) \\
4.46 \pm 2.00 \\
(3.72,5.19)\end{array}$ & $\begin{array}{l}(n=35) \\
4.18 \pm 2.14 \\
(3.45,4.92)\end{array}$ & \\
\hline
\end{tabular}

Values are mean \pm standard deviation ( $95 \%$ confidence interval)

*ANOVA $p$ value for the interaction effect of the subgroup $\times$ treatment group
$>4 \mathrm{~h}$ in the home therapy and control groups (Table 4). Respiratory values were also similar in both groups and indicated good control of sleep-disordered breathing (Table 3). Sleepiness and sleep quality at 6 months were not significantly different in the two groups (Table 5). Mean time to initiation of APAP therapy was significantly shorter in the home therapy group ( $7.6 \pm 7.2$ days, range $0-40$ days) compared with the control group (46.1 \pm 23.8 days, range $1-119$ days), $p<$ 0.0001 .

\section{Discussion}

This study found that device usage, control of sleepdisordered breathing and sleep quality as well as quality of life did not differ when sleep physicians managed APAP therapy at home with ongoing telemonitoring compared with standard in-laboratory, PSG-guided management. Results showed a larger variation in device usage time than expected in the initial sample size calculation. Due to this large variation, the initially defined non-inferiority target of $-0.3 \mathrm{~h} /$ day could not be proven (Table 2). However, the mean usage in the home therapy was $0.06 \mathrm{~h} /$ night higher than in the standard of care group, suggesting that the two approaches were associated with clinically similar levels of APAP device usage after 6 months, despite the fact that statistical significance based on the initially pre-defined NIM of $-0.3 \mathrm{~h} /$ night was not achieved. A between-group mean difference in a device usage of $>0.5 \mathrm{~h} /$ night has been defined as clinically significant [25], and non-inferiority was demonstrated in the PP population using $-0.5 \mathrm{~h} /$ night as the NIM.

In both groups, changes from baseline in the ESS and FOSQ scores also exceeded thresholds defined as being 
Table 4 Device usage and respiratory parameters at 6 months (secondary endpoints; per-protocol population)

\begin{tabular}{|c|c|c|c|}
\hline & Home therapy group & Control group & $p$ value \\
\hline Device data at 6 months & $(n=89)$ & $(n=93)$ & \\
\hline Total days used, $\%$ & $\begin{array}{l}79 \pm 24 \\
(73.6,83.7)\end{array}$ & $\begin{array}{l}74 \pm 29 \\
(68,79)\end{array}$ & 0.19 \\
\hline Days with usage $>4 \mathrm{~h}, \%$ & $\begin{array}{l}61 \pm 30 \\
(55,67)\end{array}$ & $\begin{array}{l}60 \pm 33 \\
(52,67)\end{array}$ & 0.75 \\
\hline Respiratory events, /h & $\begin{array}{l}1.8 \pm 1.9 \\
(1.4,2.2)\end{array}$ & $\begin{array}{l}2.0 \pm 2.3 \\
(1.5,2.4)\end{array}$ & 0.55 \\
\hline $95 \%$ leakage, $\mathrm{L} / \mathrm{min}$ & $\begin{array}{l}15.6 \pm 11.6 \\
(13.1,18.0)\end{array}$ & $\begin{array}{l}17.9 \pm 35.0 \\
(10.7,25.1)\end{array}$ & 0.54 \\
\hline AHI, /h & $\begin{array}{l}3.7 \pm 4.6 \\
(2.8,4.7)\end{array}$ & $\begin{array}{l}5.1 \pm 8.6 \\
(3.4,6.9)\end{array}$ & 0.17 \\
\hline $\mathrm{ODI}, / \mathrm{h}$ & $\begin{array}{l}3.5 \pm 4.7 \\
(2.5,4.5)\end{array}$ & $\begin{array}{l}5.7 \pm 10.3 \\
(3.6,7.9)\end{array}$ & 0.06 \\
\hline $\mathrm{OAI}, / \mathrm{h}$ & $\begin{array}{l}6.0 \pm 14.6 \\
(2.9,9.1)\end{array}$ & $\begin{array}{l}6.2 \pm 17.9 \\
(2.5,9.9)\end{array}$ & 0.94 \\
\hline $\mathrm{CAI}, / \mathrm{h}$ & $\begin{array}{l}3.4 \pm 5.5 \\
(2.2,4.5)\end{array}$ & $\begin{array}{l}4.9 \pm 10.2 \\
(2.8,7.1)\end{array}$ & 0.20 \\
\hline $\mathrm{MAI}, / \mathrm{h}$ & $\begin{array}{l}0.4 \pm 1.3 \\
(0.1,0.6)\end{array}$ & $\begin{array}{l}0.8 \pm 3.3 \\
(0.2,1.5)\end{array}$ & 0.19 \\
\hline $\mathrm{HI}, / \mathrm{h}$ & $\begin{array}{l}20.8 \pm 32.2 \\
(13.9,27.6)\end{array}$ & $\begin{array}{l}27.0 \pm 46.0 \\
(17.5,36.5)\end{array}$ & 0.29 \\
\hline Mean $\mathrm{SpO}_{2}, \%$ & $\begin{array}{l}93 \pm 5 \\
(92,94)\end{array}$ & $\begin{array}{l}93 \pm 2 \\
(92,93)\end{array}$ & 0.73 \\
\hline Minimum $\mathrm{SpO}_{2}, \%$ & $\begin{array}{l}84 \pm 6 \\
(82,85)\end{array}$ & $\begin{array}{l}82 \pm 8 \\
(81,84)\end{array}$ & 0.21 \\
\hline Mean APAP pressure, $\mathrm{cmH}_{2} \mathrm{O}$ & $\begin{array}{l}8.3 \pm 2.2 \\
(7.8,8.7)\end{array}$ & $\begin{array}{l}8.5 \pm 2.0 \\
(8.1,8.9)\end{array}$ & 0.57 \\
\hline $95 \%$ APAP pressure, $\mathrm{cmH}_{2} \mathrm{O}$ & $\begin{array}{l}11.2 \pm 2.3 \\
(10.7,11.7)\end{array}$ & $\begin{array}{l}12.7 \pm 10.4 \\
(10.6,14.9)\end{array}$ & 0.16 \\
\hline
\end{tabular}

Values are mean \pm standard deviation ( $95 \%$ confidence interval)

$A H I$ apnea-hypopnea index, $C A I$ central apnea index, $H I$ hypopnea index, $M A I$ mixed apnea index, $O A I$ obstructive apnea index, $O D I$ oxygen desaturation index clinically significant [25], highlighting the effectiveness of CPAP therapy in OSA from a patient perspective. It should also be noted that patients in the home therapy group received telemedicine care from the homecare provider throughout the entire study period. This is important because limiting telemedicine-based care (e.g. to 90 days) is associated with a reduction in compliance as has been shown previously [26].

Our study systematically evaluated home therapy with APAP managed by sleep physicians in Germany for the first time. It was also the first time that telemedical care was

Table 5 Change from baseline to 6 months in sleepiness and sleep quality (for all patients who had both baseline and 6-month data available)

\begin{tabular}{llll}
\hline & Home therapy group & Control group & Between-group difference in change from baseline \\
\hline ESS score & $(\mathrm{n}=90)$ & $(\mathrm{n}=92)$ & $0.7 \pm 4.5$ \\
& $-2.8 \pm 4.4$ & $-3.5 \pm 4.7$ & $(-0.6,2.0)$ \\
PSQI score & $(-3.7,-1.9)$ & $(-4.5,-2.5)$ & \\
& $(\mathrm{n}=90)$ & $(\mathrm{n}=93)$ & $0.1 \pm 4.6$ \\
& $-2.4 \pm 5.6$ & $-2.5 \pm 3.4$ & $(-1.3,1.4)$ \\
FOSQ score & $(-3.6,-1.3)$ & $(-3.2,-1.8)$ & \\
& $(\mathrm{n}=90)$ & $(\mathrm{n}=89)$ & $4.7 \pm 23.3$ \\
& $10.7 \pm 22.1$ & $6.1 \pm 24.5$ & $(-2.2,11.5)$ \\
\hline
\end{tabular}

Values are mean \pm standard deviation (95\% confidence interval) 
performed in this setting. Similar outcomes in terms of sleepiness and quality of life with APAP care provided in the home setting versus sleep laboratories have been reported in a metaanalysis [27], consistent with our findings.

One interesting finding of the study was that the time to therapy was significantly shorter using the home-based therapy initiation pathway, which has been reported previously [28]. In our study, patients in the home therapy group received APAP therapy initiation, on average, more than a month sooner than those in the control group, who went to a sleep lab for up to 2 subsequent PSG nights for treatment initiation, in line with the German standards for OSA therapy. The clinical relevance of this remains to be determined. However, earlier diagnosis and therapy of OSA should be beneficial for patients in terms of alleviating OSA symptoms and any potential negative health consequences associated with sleep-disordered breathing. The decrease in waiting times was even greater (up to 6 months) in another recent study that compared a home- versus hospital-based approach to the management of suspected OSA and found the ambulatory approach to be noninferior to hospital management [28]. The home-based management group also showed a significant improvement in the Sleep Apnoea Quality of Life Index score compared with the standard hospital approach, suggesting that the ambulatory option was better for patients in terms of improved diseasespecific quality of life. Another important finding of the study was substantially lower mean costs per patient in the homeversus hospital-based group (HK $\$ 8479$ vs. HK $\$ 22,248$ ) [28]. In addition to the savings achieved by eliminating PSG, both diagnostic and therapeutic, it has also been suggested that nursing time is reduced when a telemonitoring approach is used [29].

There is a growing body of evidence suggesting that PG can be used instead of PSG for the diagnosis and management of OSA. In a study conducted at seven AASM-accredited sleep centers, a home- and PG-based strategy for the diagnosis of OSA and titration/management of APAP therapy was not inferior to in-laboratory PSG with respect to acceptance, adherence, time to treatment and functional improvements at 1 and 3 months [30]. Several other studies have reported similar findings that point towards viability and non-inferiority of the ambulatory treatment initiation [31-36].

The ability of home-based diagnosis and management of OSA holds the potential to improve the efficiency of OSA management, both in terms of healthcare costs and improving access to care [24, 28]. From a US payer perspective, use of PG and APAP has been shown to be significantly less costly than in-lab sleep study and PSG titration [21]. In addition, implementation of a sleep telemedicine protocol improved management of sleep apnea over a period of 5 years, despite an increased demand for services [24]. Data from other studies have also highlighted cost benefits with the use of home respiratory PG management compared with PSG, with similar effectiveness and equivalent or better adherence [20, 36]. It has been suggested that PSG is probably not necessary for the majority of patients with suspected sleep apnea, with a clear economic benefit from the use of home-based management approaches [36]. Nevertheless, it is important to note that the ambulatory approach in our study was managed by sleep physicians and that it is not suitable for all OSA patients. All studies in this field have a variety of exclusion criteria, including unstable comorbidity, other sleep disorders, central sleep apnea, mental illness, drugs, pretreated sleep apnea and others. Therefore, the need for further diagnostic assessment by a certified sleep physician including PSG remains for patients who meet these criteria.

Overall, our findings demonstrate the non-inferiority of the home therapy approach for the diagnosis and management of moderate and severe OSA (AHI $>15 / \mathrm{h})$. The results point to a number of potential advantages, both for the healthcare system $[21,28,36]$ and for patients (e.g. faster access to care) [28]. In addition to supporting these existing data, our study extends previous work because we used a follow-up period of 6 months, at least twice as long as in earlier studies.

Several limitations of our study can be pointed out:

By applying an AHI of $>15 / \mathrm{h}$ as inclusion criterion, patients with mild OSA were not included in the investigation. This should be taken into account when applying these results to the general population. The mild OSA group represents a significant part of the symptomatic OSA population and therefore should be addressed in future investigations.

The study design was aimed to demonstrate non-inferiority of a home-based approach in comparison to conventional sleep lab-based APAP treatment initiation with regards to treatment adherence; it did not specifically investigate differences in cost, workload or other measures between the groups. The workload during the 6 months of telemetric follow-up for the home therapy group was not precisely measured but was retrospectively estimated at up to $2 \mathrm{~h}$ per patient-month. Meanwhile, the sleep lab group did not receive any structured follow-up after their initial therapy initiation of up to 2 consecutive PSG nights. Future studies are encouraged to investigate details of potential cost reductions and efficacy gains associated with home-based therapy of OSA.

In conclusion, use of a home-based telemonitoring strategy for initiation of APAP therapy by sleep physicians in selected patients with OSA is feasible and comparable to a standard approach with respect to device usage, respiratory parameters, and sleep quality, as well as disease-specific quality of life after 6 months of therapy. In addition, the home-based approach may facilitate faster and easier access to diagnosis and effective therapy in patients with suspected OSA.

Acknowledgements Medical writing assistance was provided by Nicola Ryan, independent medical writer, funded by ResMed. The authors 
would like to thank Ms. Maria Maass and Ms. Daniela Linke from ASR (Advanced Sleep Research $\mathrm{GmbH}$ ) for their support in carrying out the study.

Funding Open Access funding enabled and organized by Projekt DEAL. This study was funded by ResMed. TP was partially supported by a grant from Russian Federation RF Government grant no. 075-15-2019-1885.

\section{Declarations}

Ethics approval All procedures performed in studies involving human participants were in accordance with the ethical standards of the institutional and/or national research committee and with the 1964 Helsinki Declaration and its later amendments or comparable ethical standards.

Consent to participate Informed consent was obtained from all individual participants included in the study.

Conflict of interest TP holds shares of Advanced Sleep Research $\mathrm{GmbH}$, Somnico GmbH, The Siesta Group GmbH, Nukute. TP is or was consultant to Bayer, Jazz Pharma, Nukute. TP received unrestricted research grants from Cidelec, Itamar, Löwenstein Medical, Philips.

Open Access This article is licensed under a Creative Commons Attribution 4.0 International License, which permits use, sharing, adaptation, distribution and reproduction in any medium or format, as long as you give appropriate credit to the original author(s) and the source, provide a link to the Creative Commons licence, and indicate if changes were made. The images or other third party material in this article are included in the article's Creative Commons licence, unless indicated otherwise in a credit line to the material. If material is not included in the article's Creative Commons licence and your intended use is not permitted by statutory regulation or exceeds the permitted use, you will need to obtain permission directly from the copyright holder. To view a copy of this licence, visit http://creativecommons.org/licenses/by/4.0/.

\section{References}

1. Franklin KA et al (2013) Sleep apnoea is a common occurrence in females. Eur Respir J 41(3):610-615

2. Heinzer R et al (2015) Prevalence of sleep-disordered breathing in the general population: the HypnoLaus study. Lancet Respir Med 3(4):310-318

3. Fietze I et al (2018) Prevalence and association analysis of obstructive sleep apnea with gender and age differences - results of SHIPTrend. J Sleep Res:e12770

4. Bradley TD, Floras JS (2009) Obstructive sleep apnoea and its cardiovascular consequences. Lancet 373(9657):82-93

5. Mokhlesi B et al (2014) Obstructive sleep apnea during REM sleep and hypertension. results of the Wisconsin Sleep Cohort. Am J Respir Crit Care Med 190(10):1158-1167

6. Lavergne $\mathrm{F}$ et al (2015) Atrial fibrillation and sleep-disordered breathing. J Thorac Dis 7(12):E575-E584

7. Gilat $\mathrm{H}$ et al (2014) Obstructive sleep apnea and cardiovascular comorbidities: a large epidemiologic study. Medicine (Baltimore) 93(9):e45

8. Lipford MC et al (2015) Associations between cardioembolic stroke and obstructive sleep apnea. Sleep 38(11):1699-1705

9. Kent BD et al (2014) Diabetes mellitus prevalence and control in sleep-disordered breathing: the European Sleep Apnea Cohort (ESADA) study. Chest 146(4):982-990
10. Martinez-Garcia MA, Campos-Rodriguez F, Barbe F (2016) Cancer and OSA: current evidence from human studies. Chest $150(2): 451-463$

11. Epstein LJ et al (2009) Clinical guideline for the evaluation, management and long-term care of obstructive sleep apnea in adults. J Clin Sleep Med 5(3):263-276

12. Weaver TE, Grunstein RR (2008) Adherence to continuous positive airway pressure therapy: the challenge to effective treatment. Proc Am Thorac Soc 5(2):173-178

13. Kohler M et al (2010) Predictors of long-term compliance with continuous positive airway pressure. Thorax 65(9):829-832

14. Weaver TE, Sawyer AM (2010) Adherence to continuous positive airway pressure treatment for obstructive sleep apnoea: implications for future interventions. Indian J Med Res 131:245-258

15. Meslier N et al (1998) A French survey of 3,225 patients treated with CPAP for obstructive sleep apnoea: benefits, tolerance, compliance and quality of life. Eur Respir J 12(1):185-192

16. Wells RD et al (2007) Adherence, reports of benefits, and depression among patients treated with continuous positive airway pressure. Psychosom Med 69(5):449-454

17. Hoffstein V et al (1992) Treatment of obstructive sleep apnea with nasal continuous positive airway pressure. Patient compliance, perception of benefits, and side effects. Am Rev Respir Dis 145(4 Pt 1):841-845

18. Flemons WW et al (2004) Access to diagnosis and treatment of patients with suspected sleep apnea. Am J Respir Crit Care Med 169(6):668-672

19. Alonso-Alvarez ML et al (2015) Reliability of home respiratory polygraphy for the diagnosis of sleep apnea in children. Chest 147(4):1020-1028

20. Andreu AL et al (2012) Effect of an ambulatory diagnostic and treatment programme in patients with sleep apnoea. Eur Respir J 39(2):305-312

21. Kim RD et al (2015) An economic evaluation of home versus laboratory-based diagnosis of obstructive sleep apnea. Sleep 38(7):1027-1037

22. Masa JF et al (2011) Effectiveness of home respiratory polygraphy for the diagnosis of sleep apnoea and hypopnoea syndrome. Thorax 66(7):567-573

23. Pinna GD et al (2014) Can cardiorespiratory polygraphy replace portable polysomnography in the assessment of sleep-disordered breathing in heart failure patients? Sleep Breath 18(3):475-482

24. Baig MM, Antonescu-Turcu A, Ratarasarn K (2016) Impact of sleep telemedicine protocol in management of sleep apnea: a 5year VA experience. Telemed J E Health 22(5):458-462

25. Kapur VK et al (2017) Clinical practice guideline for diagnostic testing for adult obstructive sleep apnea: an American Academy of Sleep Medicine Clinical Practice Guideline. J Clin Sleep Med 13(3):479-504

26. Hwang D et al (2018) Effect of telemedicine education and telemonitoring on continuous positive airway pressure adherence. The Tele-OSA Randomized Trial. Am J Respir Crit Care Med 197(1):117-126

27. Kunisaki KM et al. (2016) VA Evidence-based synthesis program reports, in The comparative effectiveness, harms, and cost of care models for the evaluation and treatment of obstructive sleep apnea (OSA): a systematic review, Department of Veterans Affairs (US): Washington (DC).

28. Hui DS et al (2017) A randomized controlled trial of an ambulatory approach versus the hospital-based approach in managing suspected obstructive sleep apnea syndrome. Sci Rep 8:45901

29. Anttalainen U et al (2016) Telemonitoring of CPAP therapy may save nursing time. Sleep Breath 20(4):1209-1215

30. Rosen CL et al (2012) A multisite randomized trial of portable sleep studies and positive airway pressure autotitration versus laboratory- 
based polysomnography for the diagnosis and treatment of obstructive sleep apnea: the HomePAP study. Sleep 35(6):757-767

31. Berry RB, Sriram P (2014) Auto-adjusting positive airway pressure treatment for sleep apnea diagnosed by home sleep testing. J Clin Sleep Med 10(12):1269-1275

32. Skomro RP et al (2010) Outcomes of home-based diagnosis and treatment of obstructive sleep apnea. Chest 138(2):257-263

33. Mulgrew AT et al (2007) Diagnosis and initial management of obstructive sleep apnea without polysomnography: a randomized validation study. Ann Intern Med 146(3):157-166

34. Kuna ST et al (2011) Noninferiority of functional outcome in ambulatory management of obstructive sleep apnea. Am J Respir Crit Care Med 183(9):1238-1244
35. Antic NA et al (2009) A randomized controlled trial of nurse-led care for symptomatic moderate-severe obstructive sleep apnea. Am J Respir Crit Care Med 179(6):501-508

36. Corral J et al (2017) Conventional polysomnography is not necessary for the management of most patients with suspected obstructive sleep apnea. Noninferiority, Randomized controlled trial. Am J Respir Crit Care Med 196(9):1181-1190

Publisher's note Springer Nature remains neutral with regard to jurisdictional claims in published maps and institutional affiliations. 